\title{
Moderate coffee consumption is inversely associated with the metabolic syndrome in the Korean adult population
}

\author{
Youngyo Kim and Youjin Je* \\ Department of Food and Nutrition, Kyung Hee University, 26 Kyunghee-daero, Dongdaemun-gu, Seoul O2447, South Korea \\ (Submitted 15 February 2018 - Final revision received 6 August 2018 - Accepted 22 August 2018 - First published online 12 October 2018)
}

\section{Abstract}

Results from studies investigating the association between coffee consumption and the metabolic syndrome in Koreans have been inconsistent and remain controversial. We examined coffee intake in relation to the metabolic syndrome using data from the Korea National Health and Nutrition Examination Survey 2012-2015, with 8387 adults aged 19-64 years. Furthermore, we conducted a stratified analysis by obesity status (BMI $<25 v . \geq 25 \mathrm{~kg} / \mathrm{m}^{2}$ ) to examine whether the association varied by obesity status. A 112 -item FFQ was used to assess diet. Multivariable logistic regression models were used to determine the association of coffee consumption $(<1,1-2,3-4$ and $\geq 5$ times/d) and the metabolic syndrome. Compared to people who consumed $<1$ time/d of coffee, the OR for the metabolic syndrome in those who consumed 3-4 times/d of coffee was 0.75 (95\% CI $0.58,0.97)$. However, $\geq 5$ times/d of coffee consumption was not significantly associated with the metabolic syndrome. By obesity status, in non-obese people, those consuming more coffee had lower odds of the metabolic syndrome, showing a significant linear trend ( $P$-trend $=0.0248$ ). In obese people, the OR for the metabolic syndrome in people with $3-4$ times/d of coffee consumption $v .<1$ time/d was 0.68 (95\% CI 0.50, 0.93), but $\geq 5$ times/d of coffee consumption showed a non-significant positive association. Our findings suggest that moderate coffee consumption of 3-4 times/d is inversely associated with the metabolic syndrome in Korean adults. The association between heavy coffee consumption of $\geq 5$ times/d and the metabolic syndrome varied by obesity status.

Key words: Coffee: Metabolic syndrome: Korean adults: Obesity

The metabolic syndrome is defined as a cluster of metabolic disturbances, including abdominal obesity, dyslipidaemia (high TAG levels, low HDL-cholesterol), hyperglycaemia, and high blood pressure, which have been considered as risk factors of CVD and type 2 diabetes $^{(1)}$. The metabolic syndrome was positively associated with the risk of common cancers such as colorectal and breast cancer ${ }^{(2)}$ as well as $\mathrm{CVD}^{(3,4)}$ and diabetes $^{(3)}$. The increasing trends in the prevalence of the metabolic syndrome are evident over recent decades worldwide $^{(5)}$, and especially in Korea, the metabolic syndrome has emerged as an important public health issue, because the prevalence of the metabolic syndrome has increased significantly in Korean adults recently ${ }^{(6)}$. Dietary habits such as high red meat or soft drink consumption were considered as major risk factors of the metabolic syndrome along with several lifestyle factors including smoking, alcohol consumption and physical $\operatorname{activity}^{(1,7,8)}$, and several meta-analyses examined the association between food intake and the metabolic syndrome ${ }^{(7-11)}$.

Coffee is one of the most consumed beverages throughout the world. Recently, increasing epidemiological studies investigated the association between coffee consumption and the metabolic syndrome, and suggested the possible beneficial effects of coffee ${ }^{(10,12)}$. However, studies targeting the Korean population have shown conflicting results on this topic recently. One study suggested protective effects of coffee consumption on the metabolic syndrome in women ${ }^{(13)}$, while another study reported that high coffee intake may increase the risk of the metabolic syndrome ${ }^{(14)}$. The other study did not find a significant association between coffee consumption and the metabolic syndrome in Korean adults ${ }^{(15)}$. As the average frequency of coffee consumption among Korean adults is high as 11.3 times per week recently ${ }^{(16)}$, it is important to examine the association between coffee consumption and the metabolic syndrome in Korean adults. Furthermore, some of the coffee drinkers put additives such as sugar and cream in coffee, and especially in Korea, relatively high percentages of coffee drinkers consume coffee with sugar and cream ${ }^{(17,18)}$. Coffee additives such as sugar and cream may increase the risk of obesity $^{(19,20)}$, and there were some studies which reported that the positive association between intakes of added sugars and the metabolic syndrome was more evident in obese people, compared to non-obese people ${ }^{(21,22)}$. To our knowledge, there

Abbreviation: KNHANES, Korea National Health and Nutrition Examination Survey.

* Corresponding author: Y. Je, fax +82 2961 0538, email youjinje@khu.ac.kr 
were few studies which investigated the relationship between coffee consumption and the metabolic syndrome in subjects stratified by obesity status.

Therefore, we aimed to assess the prevalence of the metabolic syndrome according to coffee consumption in general Korean population using data from nationally representative surveys in this study. In addition, we conducted a stratified analysis by obesity status to examine whether the association between coffee and the metabolic syndrome differs according to obesity status. We hypothesised that moderate coffee consumption was inversely associated with the metabolic syndrome and the association varied by obesity status.

\section{Methods}

\section{Study population}

The present study was based on data from the Korea National Health and Nutrition Examination Survey (KNHANES), which was released by Korea Centers for Disease Control and Prevention under Korean Ministry of Health and Welfare. The KNHANES is the nationally representative cross-sectional survey of the South Korean population ${ }^{(23)}$. The KNHANES has been conducted annually to gather information on health related behaviours, health conditions, and nutritional states of Koreans through three surveys including a health interview, a health examination, and a nutrition survey. The KNHANES used a clustered, multistage, stratified and probability sampling design based on age-groups, sex and geographical region to represent the non-institutionalised civilian South Korean population. More details of the data-collection/physical examination method of KNHANES have been previously reported ${ }^{(23)}$. We collected data from 2012 ( $n$ 8058), 2013 ( $n$ 8018), 2014 ( $n$ 7550) and 2015 ( $n$ 7380), which summed up to a total of 31006 participants. Among the participants, 26263 subjects who completed the health interview, health examination and nutrition survey were included. Then, we sequentially excluded the following individuals who were not eligible for this study: 11459 subjects with age of $<19$ or $\geq 65$ years who did not report dietary intakes using the FFQ; 3509 subjects with a self-reported diagnosis of diabetes, hypertension, dyslipidaemia, myocardial infarction, stroke or cancer, or were taking medications to treat diabetes, dyslipidaemia or hypertension; ninety-six subjects who were pregnant; 678 subjects who had missing responses on HDL-cholesterol, TAG, fasting serum glucose, and systolic and diastolic blood pressure; ninety-seven subjects with no fasting status ( $<8 \mathrm{~h}$ ) at blood test; 859 subjects who had extreme energy intake ( $\leq 2093$ or $>25113 \mathrm{~kJ} / \mathrm{d})$; subjects with missing information on moderate or vigorous physical activity ( $n$ 182) and alcohol consumption ( $n$ 996). In summary, the final analysis included 8387 subjects (2275 from 2012, 2233 from 2013, 1962 from 2014 and 1917 from 2015) including 3359 men and 5028 women. A flow diagram of the selection for study population is shown in online Supplementary Fig. S1. Each participant in the survey submitted informed consent and the Institutional Review Board of Korea Centers for Disease Control and Prevention provided formal ethics approval for the
KNHANES data sets (IRB no. 2012-01EXP-01-2C, 2013-07CON-03-4C, 2013-12EXP-03-5C, 2015-01-02-6C).

\section{Dietary assessment}

Coffee consumption was collected using the 112-item dish-based FFQ of KNHANES. The reproducibility and validity of the FFQ have already been evaluated through 3 -d diet records elsewhere ${ }^{(24)}$. Subjects were asked to select from nine categories of coffee consumption frequency over the last year, ranging from 'almost never' to ' 3 times per day'. The amount of the coffee additives such as cream and sugar was also collected. We considered the intakes of other food groups including green tea, soda, red meat, white meat, vegetables, fruit, fish, dairy products, nuts and whole grains as potential confounding variables. The serving size were composed of three categories (a half of, equal to, and 1.5 or 2 times of a standard serving size). The details of food groups are presented in online Supplementary Table S1.

\section{Assessment of the metabolic syndrome}

Waist circumference was measured by trained researchers to the nearest $0 \cdot 1 \mathrm{~cm}$ as the abdominal girth midway between the iliac crest and the lowest rib with each subject's weight distributed evenly on both feet at the end of a normal expiration. Health professionals measured blood pressure three times after subjects had rested for $5 \mathrm{~min}$ in a sitting posture. The average of the last two readings for systolic blood pressure and diastolic blood pressure was used for analysis. The serum levels of glucose, TAG, and cholesterol were measured enzymatically using a Hitachi automatic analyzer 7600 (Hitachi) in 2012 and COBAS 8000 C702 (Roche) in 2013, 2014 and 2015 at the Neodin Medical Institute in Korea. The definition of the metabolic syndrome came from the harmonising criteria by Alberti et $a l .{ }^{(3)}$. Participants have been considered to have the metabolic syndrome when they have at least three of the following criteria: (1) abdominal obesity (waist circumference $\geq 90 \mathrm{~cm}$ for men and $\geq 80 \mathrm{~cm}$ for women); (2) low HDL-cholesterol (HDLcholesterol $<1.0 \mathrm{mmol} / \mathrm{l}$ for men and $<1.3 \mathrm{mmol} / 1$ for women); (3) hypertriacylglycerolaemia (TAG $\geq 1.7 \mathrm{mmol} / \mathrm{l}$ ); (4) hyperglycaemia (fasting plasma glucose $\geq 5.6 \mathrm{mmol} / \mathrm{l}$ ) and (5) elevated blood pressure (systolic blood pressure/diastolic blood pressure $\geq 130 / 85 \mathrm{mmHg}$ ).

\section{Demographic and lifestyle factors}

The demographic factors including age, sex and socioeconomic status were obtained using a self-administered questionnaire. Education level was categorised into 'elementary school or lower', 'middle or high school' and 'college or higher', and household income was divided into 'lowest', 'lower middle', 'upper middle' and 'highest'. The behavioural information including alcohol consumption, physical activity and smoking status was also self-reported. The frequency and the amount of alcohol drinking yearly were collected, and we evaluated the weekly servings of alcoholic beverages via multiplying the frequency of alcohol drinking by the amount of alcoholic beverage. Alcohol consumption was categorised into $<1,1-2$ and $\geq 3$ 
servings/d. Physical activity was assessed by the practice of aerobic activities. Participants who have $\geq 75 \mathrm{~min} /$ week of vigorous activity, $\geq 150 \mathrm{~min} /$ week of moderate activity or $\geq 150 \mathrm{~min} /$ week of a combination of moderate and vigorous activity ( $1 \mathrm{~min}$ of vigorous activity was considered equal to $2 \mathrm{~min}$ of moderate activity) were considered physically active. Smoking status was classified as current smoker, non-smoker and ex-smoker.

\section{Statistical analysis}

Coffee consumption frequency was divided into four categories ( $<1,1-2,3-4$ and $\geq 5$ times/d). The age-standardised prevalence and mean of demographic factors and food intakes were estimated in each coffee consumption category using the PROC SURVEYLOGISTIC and PROC SURVEYREG (SAS Institute, Cary, NC, USA) procedures considering the clustering sampling design of the survey. We used the PROC SURVEYLOGISTIC procedure to assess the association between coffee consumption and endpoints (the metabolic syndrome and each component of the metabolic syndrome). We applied a sample weighting in the analysis using WEIGHT code in the PROC SURVEY procedure. We developed two different models which adjusted for various confounders. Model 1 adjusted for age (continuous), sex, BMI $\left(\mathrm{kg} / \mathrm{m}^{2}\right.$, continuous), total energy intake (kcal, continuous), household income (lowest, lower middle, upper middle and highest), education (elementary school or lower, middle or high school and college or higher), alcohol consumption ( $<1,1-2$ and $\geq 3$ servings/d), smoking (non-smoker, ex-smoker and current smoker), physical activity (inactive and active) and survey year (2012, 2013, 2014 and 2015). Model 2 further adjusted for sleep duration $(<6,6-<8$ and $\geq 8 \mathrm{~h} / \mathrm{d})$ and several dietary factors such as soda, green tea, red meat, white meat, vegetables, fruit, fish, dairy products, legumes, nuts and whole grains (continuous, servings/week). When we chose covariates for multivariable models, we referred the results of analysis for characteristics of study subjects by coffee consumption and previous literature using the KNHANES data ${ }^{(25,26)}$ or reporting the association between food consumption and the metabolic syndrome ${ }^{(27,28)}$. The PROC SURVEYLOGISTIC procedure was used to examine a linear trend across coffee consumption categories by using the median value within each exposure category. We conducted the stratified analysis by obesity status (BMI $<25 v$. $\geq 25 \mathrm{~kg} / \mathrm{m}^{2}$ ). All statistical analyses were performed with SAS software (version 9.4; SAS Institute). A two-tailed $P$ value $<0.05$ was considered to be statistically significant.

\section{Results}

\section{Baseline characteristics}

The basic characteristics of the participants by the coffee consumption are shown in Table 1. People in the highest category of coffee consumption ( $\geq 5$ times/d) were more likely to be older and men, and to have higher BMI and education level, and shorter sleep hours, compared with those in the lowest category $(<1$ time/d). Participants who consumed more coffee had higher alcohol consumption and energy intake, and were more likely to be current smokers. Frequent coffee consumers tended to consume more coffee additives such as cream and sugar. Coffee consumption was inversely associated with the intakes of fruit, whole grain and dairy products.

Table 2 shows the characteristics of study subjects stratified by obesity status. Subjects with BMI $\geq 25 \mathrm{~kg} / \mathrm{m}^{2}$ were more likely to be older, men, current smokers, and physically active, and to have higher alcohol consumption and lower education level, family incomes, and sleep hours than those with BMI $<25 \mathrm{~kg} / \mathrm{m}^{2}$. Food consumption including coffee was mostly higher in obese people than non-obese people, except for fruit.

\section{Coffee consumption and the metabolic syndrome}

The results of multivariable analysis on the association between coffee consumption and the metabolic syndrome were presented in Table 3. After the multivariable adjustment including other dietary intakes in model 2 , participants who consumed 3-4 times/d of coffee had $25 \%$ lower odds of having the metabolic syndrome compared with those who consumed $<1$ time/d of coffee (OR $=0.75,95 \%$ CI $0 \cdot 58,0.97)$. There was no significant difference in the odds of the metabolic syndrome for five or more times per day $v .<1$ time/d (OR $=0 \cdot 90,95 \% \mathrm{CI}$ $0 \cdot 64,1 \cdot 25)$. Regarding the components of the metabolic syndrome, participants who consumed five or more times per day of coffee had 1.36 times higher odds of having low HDLcholesterol than those who consumed $<1$ time/d of coffee $(\mathrm{OR}=1 \cdot 36,95 \% \mathrm{CI} 1 \cdot 04,1 \cdot 77)$. Although all the individual OR were not significant, a significant inverse linear trend was found for the hyperglycaemia ( $P$-trend $=0 \cdot 0416$ ).

To further evaluate the association of coffee consumption and the metabolic syndrome, we conducted a stratified analysis by obesity status (Table 4). In non-obese people, five or more times per day of coffee consumption was inversely associated with the metabolic syndrome (OR $=0.47,95 \%$ CI $0 \cdot 24,0.90)$, and a significant inverse linear trend was also found ( $P$-trend $=0 \cdot 0248$ ). For obese people, those consuming 3-4 times/d of coffee had $32 \%$ lower odds of having the metabolic syndrome than subjects consuming $<1$ time/d of coffee $(\mathrm{OR}=$ $0 \cdot 68,95 \%$ CI $0.50,0.93$ ), but five or more times per day of coffee consumption showed a non-significant positive association. When we further adjusted for intake of coffee additives including sugar and cream, the association was slightly stronger among obese people consuming 3-4 times/d of coffee (OR=0.64, 95\% CI 0.44, 0.91, data not shown).

\section{Discussion}

In the present study from nationally representative survey data, we found that moderate coffee consumption was inversely associated with the metabolic syndrome. People with 3-4 times/d of coffee had $25 \%$ lower odds of having the metabolic syndrome compared with those with $<1$ time/d of coffee. In all, five or more times per day of coffee consumption, however, did not show a significant association for the metabolic syndrome. By obesity status, significantly lower odds of having the metabolic syndrome with 3-4 times/d of coffee consumption was also found among obese individuals and showed a non- 
Table 1. Characteristics of the study subjects according to coffee consumption (Mean values with their standard errors)

\begin{tabular}{|c|c|c|c|c|c|c|c|c|c|}
\hline & \multicolumn{8}{|c|}{ Coffee consumption } & \multirow[b]{3}{*}{$P^{\star}$} \\
\hline & \multicolumn{2}{|c|}{$<1$ time/d } & \multicolumn{2}{|c|}{$1-2$ times/d } & \multicolumn{2}{|c|}{ 3-4 times/d } & \multicolumn{2}{|c|}{$\geq 5$ times $/ \mathrm{d}$} & \\
\hline & Mean & SE & Mean & SE & Mean & SE & Mean & SE & \\
\hline$n$ & \multicolumn{2}{|c|}{2804} & \multicolumn{2}{|c|}{3609} & \multicolumn{2}{|c|}{1488} & \multicolumn{2}{|c|}{486} & \\
\hline Aget (years) & 33.5 & 0.3 & 39.5 & 0.2 & $42 \cdot 7$ & 0.3 & $41 \cdot 1$ & 0.5 & $<0.0001$ \\
\hline Male $(\%)$ & $48 \cdot 1$ & $1 \cdot 1$ & 44.5 & 0.9 & 63.5 & 1.4 & 80.0 & 1.9 & $<0.0001$ \\
\hline Energy intake $\neq$ (kcal/d) & $2124 \cdot 6$ & $18 \cdot 7$ & $2165 \cdot 0$ & $16 \cdot 7$ & 2324.9 & 24.4 & $2695 \cdot 3$ & $45 \cdot 7$ & $<0.0001$ \\
\hline BMI $\neq\left(\mathrm{kg} / \mathrm{m}^{2}\right)$ & $23 \cdot 3$ & 0.1 & $23 \cdot 2$ & 0.1 & $23 \cdot 7$ & 0.1 & $24 \cdot 1$ & 0.2 & $<0.0001$ \\
\hline High physical activity§॥ (\%) & 50.5 & $1 \cdot 2$ & $49 \cdot 4$ & $1 \cdot 1$ & $49 \cdot 1$ & 1.5 & 45.5 & 2.5 & 0.3295 \\
\hline Alcohol, $\geq 1$ drink/dll (\%) & $24 \cdot 3$ & 1.0 & $24 \cdot 3$ & 0.9 & $30 \cdot 3$ & 1.4 & 40.4 & 2.5 & $<0.0001$ \\
\hline High education IIII (\%) & $38 \cdot 3$ & $1 \cdot 2$ & 50.5 & 1.1 & $46 \cdot 8$ & 1.7 & 43.8 & $2 \cdot 7$ & $<0.0001$ \\
\hline Currently smokingll (\%) & $16 \cdot 9$ & 0.9 & 21.7 & 0.9 & $40 \cdot 6$ & 1.5 & $67 \cdot 0$ & 2.4 & $<0.0001$ \\
\hline Highest income quartilell (\%) & $34 \cdot 3$ & 1.2 & $37 \cdot 3$ & 1.2 & 34.7 & 1.6 & 34.2 & $2 \cdot 6$ & 0.1351 \\
\hline Sleep duration, $\geq 8 \mathrm{~h} / \mathrm{d} \| \mathrm{l}(\%)$ & $31 \cdot 7$ & $1 \cdot 1$ & $28 \cdot 4$ & 0.9 & 25.8 & 1.4 & 29.0 & 2.5 & 0.0105 \\
\hline \multicolumn{10}{|l|}{ Coffee additives } \\
\hline Cream (teaspoons/d) $\ddagger$ & 0.2 & 0.01 & 1.0 & 0.02 & 2.5 & 0.05 & 5.5 & 0.21 & $<0.0001$ \\
\hline Sugar (teaspoons/d) $\ddagger$ & 0.2 & 0.01 & 1.2 & 0.02 & $2 \cdot 7$ & 0.05 & 5.8 & 0.21 & $<0.0001$ \\
\hline \multicolumn{10}{|l|}{ Dietary intake } \\
\hline Vegetablesł (servings/week) & 17.06 & 0.29 & 17.67 & 0.27 & 17.83 & 0.41 & 19.67 & 0.75 & $<0.0001$ \\
\hline Fruitł (servings/week) & 9.58 & 0.20 & $9 \cdot 13$ & 0.16 & 8.23 & 0.23 & 8.29 & 0.38 & $<0.0001$ \\
\hline Legumesł (servings/week) & 4.56 & 0.10 & 4.50 & 0.08 & 4.52 & 0.13 & 4.78 & 0.21 & $<0.0001$ \\
\hline Whole grains $\ddagger$ (servings/week) & 8.25 & 0.15 & 7.46 & 0.13 & 6.78 & 0.20 & 5.63 & 0.36 & $<0.0001$ \\
\hline Red meatł (servings/week) & 4.50 & 0.09 & 4.98 & 0.09 & 5.55 & 0.12 & 6.96 & 0.31 & $<0.0001$ \\
\hline White meatł (servings/week) & 1.94 & 0.05 & 1.99 & 0.04 & 2.08 & 0.06 & 2.40 & 0.13 & $<0.0001$ \\
\hline Fish (servings/week) & 4.02 & 0.13 & 4.08 & $0 \cdot 12$ & $4 \cdot 11$ & 0.17 & 4.67 & 0.33 & $<0.0001$ \\
\hline Dairy products $\ddagger$ (servings/week) & 5.43 & 0.16 & $5 \cdot 34$ & 0.11 & 4.87 & 0.17 & 4.76 & 0.27 & $<0.0001$ \\
\hline Nuts $\ddagger$ (servings/week) & 0.62 & 0.03 & 0.58 & 0.03 & 0.57 & 0.04 & 0.53 & 0.07 & $<0.0001$ \\
\hline Sodał (cups/week) & $1 \cdot 17$ & 0.05 & 1.30 & 0.06 & $1 \cdot 28$ & 0.06 & 1.87 & 0.18 & $<0.0001$ \\
\hline Green tea (cups/week) & 0.99 & 0.08 & 1.17 & 0.06 & 1.41 & 0.12 & 1.30 & 0.22 & 0.0158 \\
\hline
\end{tabular}

* $P$-values were derived from the $x^{2}$ test for categorical variables and a regression model using PROC SURVEYREG procedure for a continuous variable.

$\dagger$ Values are means.

¥ Values are age-standardised means.

$\S$ High physical activity was defined as $150 \mathrm{~min} /$ week of moderate activity, $\geq 75 \mathrm{~min} /$ week of vigorous activity or $\geq 150 \mathrm{~min} /$ week of a combination of moderate and vigorous activity.

Thlues are age-standardised prevalences.

I College education or higher. 
Table 2. Characteristics of the study subjects according to obesity status (Mean values with their standard errors)

\begin{tabular}{|c|c|c|c|c|c|}
\hline & \multicolumn{4}{|c|}{ Obesity status } & \multirow[b]{3}{*}{$P^{\star}$} \\
\hline & \multicolumn{2}{|c|}{$\mathrm{BMI}<25 \mathrm{~kg} / \mathrm{m}^{2}$} & \multicolumn{2}{|c|}{$\mathrm{BMI} \geq 25 \mathrm{~kg} / \mathrm{m}^{2}$} & \\
\hline & Mean & SE & Mean & SE & \\
\hline$n$ & \multicolumn{2}{|c|}{6112} & \multicolumn{2}{|c|}{2275} & \\
\hline Age† (years) & 37.5 & $0 \cdot 2$ & $39 \cdot 4$ & 0.3 & $<0.0001$ \\
\hline Male (\%) & $46 \cdot 1$ & 0.7 & $65 \cdot 0$ & $1 \cdot 1$ & $<0.0001$ \\
\hline Energy intake $\neq$ (kcal/d) & $2153 \cdot 2$ & $12 \cdot 7$ & 2365.5 & 22.5 & $<0.0001$ \\
\hline BMI‡ $\left(\mathrm{kg} / \mathrm{m}^{2}\right)$ & 21.7 & 0.0 & $27 \cdot 7$ & 0.1 & $<0.0001$ \\
\hline High physical activity§॥ (\%) & $48 \cdot 3$ & 0.8 & $52 \cdot 4$ & $1 \cdot 3$ & 0.0046 \\
\hline Alcohol, $\geq 1$ drink/dll (\%) & $22 \cdot 7$ & 0.6 & $35 \cdot 8$ & $1 \cdot 2$ & $<0.0001$ \\
\hline High education $\uparrow \|(\%)$ & $46 \cdot 1$ & 0.9 & $42 \cdot 6$ & $1 \cdot 3$ & 0.0137 \\
\hline Currently smokingll (\%) & $22 \cdot 8$ & 0.7 & $35 \cdot 0$ & $1 \cdot 2$ & $<0.0001$ \\
\hline Highest income quartilell (\%) & $36 \cdot 9$ & $1 \cdot 1$ & $32 \cdot 3$ & 1.4 & 0.0026 \\
\hline Sleep duration, $\geq 8 \mathrm{~h} / \mathrm{d} \|(\%)$ & 29.9 & 0.7 & $27 \cdot 2$ & $1 \cdot 1$ & 0.0364 \\
\hline Coffee (times/d)‡ & 1.6 & 0.03 & 1.8 & 0.04 & $<0.0001$ \\
\hline Cream (teaspoons/d) $\ddagger$ & $1 \cdot 2$ & 0.03 & 1.4 & 0.05 & $<0.0001$ \\
\hline Sugar (teaspoons/d) $\ddagger$ & $1 \cdot 3$ & 0.03 & 1.5 & 0.05 & $<0.0001$ \\
\hline \multicolumn{6}{|l|}{ Dietary intake } \\
\hline Vegetablesł (servings/week) & $17 \cdot 11$ & $0 \cdot 19$ & $18 \cdot 90$ & 0.38 & $<0.0001$ \\
\hline Fruits $\ddagger$ (servings/week) & $9 \cdot 15$ & $0 \cdot 13$ & 8.88 & 0.21 & 0.0432 \\
\hline Legumesł (servings/week) & 4.45 & 0.06 & $4 \cdot 77$ & 0.11 & $<0.0001$ \\
\hline Whole grains $\ddagger$ (servings/week) & $7 \cdot 51$ & $0 \cdot 11$ & $7 \cdot 48$ & 0.16 & $<0.0001$ \\
\hline Red meat $\ddagger$ (servings/week) & $4 \cdot 86$ & 0.07 & $5 \cdot 51$ & 0.12 & $<0.0001$ \\
\hline White meat $\ddagger$ (servings/week) & 1.96 & 0.03 & $2 \cdot 15$ & 0.06 & $<0.0001$ \\
\hline Fish $\ddagger$ (servings/week) & 4.04 & 0.09 & $4 \cdot 26$ & 0.14 & $<0.0001$ \\
\hline Dairy products $\ddagger$ (servings/week) & $5 \cdot 23$ & $0 \cdot 10$ & $5 \cdot 31$ & 0.15 & $<0.0001$ \\
\hline Nutsł (servings/week) & 0.58 & 0.02 & 0.62 & 0.03 & $<0.0001$ \\
\hline Sodał (cups/week) & $1 \cdot 17$ & 0.04 & 1.57 & 0.08 & $<0.0001$ \\
\hline Green teał (cups/week) & $1 \cdot 10$ & 0.05 & $1 \cdot 32$ & 0.09 & 0.0631 \\
\hline \multicolumn{6}{|l|}{$\begin{array}{l}P \text {-values were derived from the } x^{2} \text { test for } \\
\text { Values are means. } \\
\text { Values are age-standardised means. } \\
\text { \$ High physical activity was defined as } 150 \mathrm{mi} \\
\text { vigorous activity. } \\
\text { Values are age-standardised prevalences. } \\
\text { I College education or higher. }\end{array}$} \\
\hline
\end{tabular}

significant positive association with five or more times per day of coffee. On the other hand, in non-obese people, those consuming more coffee had lower odds of the metabolic syndrome, showing a significant linear trend.

The recent dose-response meta-analysis including thirteen observational studies showed that the risk of the metabolic syndrome decreased with the increase of coffee consumption, but the observed inverse association was attenuated from 5 cups/d of coffee consumption ${ }^{(9)}$. This non-linear relationship was also found for other health outcomes besides the metabolic syndrome. Another meta-analysis of thirty-six prospective studies observed a non-linear association between coffee intake and risk of CVD, showing the largest risk reduction at 3-5 cups/d of coffee consumption ${ }^{(29)}$. For all-cause mortality, the largest decrease was found at 3-4 cups/d of coffee consumption ${ }^{(30)}$ or at $2-4 \mathrm{cups} / \mathrm{d}$ of coffee consumption ${ }^{(31)}$ from two separate meta-analyses, suggesting that heavy coffee consumption is not associated with further decrease in risk of total mortality compared with moderate coffee consumption. These findings are similar to our results in that they suggested the greater protective effect of coffee consumption at a certain level of intake. There is consistent evidence that coffee consumption is generally associated with a number of health outcomes in a J-shaped manner ${ }^{(32)}$. Regarding components of the metabolic syndrome, we observed that high coffee consumption was associated with lower HDL-cholesterol and fasting plasma glucose levels. A previous study from Korean adults reported that instant coffee consumers had a 19\% higher prevalence of low HDL-cholesterol than non-consumers ${ }^{(14)}$. The results from Italy found that people consuming more coffee had lower fasting plasma glucose levels ${ }^{(33)}$.

There are several possible mechanisms which suggested that coffee might reduce the metabolic syndrome. Oxidative stress is related to the development of metabolic disorders and chronic inflammation. Coffee contains strong antioxidant components such as chlorogenic acid or melanoidins. Chlorogenic acid may reduce plasma glucose levels, hepatic glucose production, and fatty acid synthesis by inhibiting the glucose-6-phosphate translocase and activating AMP-activated protein kinase ${ }^{(34,35)}$. Also chlorogenic acid has anti-hypertensive effects through increasing nitric oxide-mediated vasodilation and ameliorating endothelial function ${ }^{(36)}$, and exhibits anti-obesity property by elevating adiponectin levels ${ }^{(37)}$. Melanoidins, which were produced during the roasting of coffee beans, may reduce blood pressure and chronic inflammation by inhibiting the angiotensin-I converting enzyme and increasing of antiinflammatory mediators ${ }^{(38)}$. However, further studies examining the association between coffee consumption and the 
Table 3. Multivariable adjusted risk for the metabolic syndrome and its individual components (Odds ratios and $95 \%$ confidence intervals)

\begin{tabular}{|c|c|c|c|c|c|c|c|c|c|}
\hline \multirow[t]{2}{*}{ Coffee consumption } & \multicolumn{2}{|c|}{$<1$ time/d } & \multicolumn{2}{|c|}{$1-2$ times/d } & \multicolumn{2}{|c|}{ 3-4 times/d } & \multicolumn{2}{|c|}{$\geq 5$ times $/ \mathrm{d}$} & \multirow[b]{2}{*}{$P$-trend ${ }^{*}$} \\
\hline & OR & $95 \% \mathrm{Cl}$ & OR & $95 \% \mathrm{Cl}$ & OR & $95 \% \mathrm{Cl}$ & OR & $95 \% \mathrm{Cl}$ & \\
\hline No. of subjects & \multirow{2}{*}{\multicolumn{2}{|c|}{2804}} & \multirow{2}{*}{\multicolumn{2}{|c|}{3609}} & \multicolumn{2}{|c|}{1488} & \multirow{2}{*}{\multicolumn{2}{|c|}{486}} & \\
\hline Metabolic syndrome & & & & & & & & & \\
\hline No. of cases & \multicolumn{2}{|c|}{372} & \multicolumn{2}{|c|}{576} & \multicolumn{2}{|c|}{253} & \multicolumn{2}{|c|}{99} & \\
\hline Age- and sex-adjusted & 1.0 & Ref. & 1.05 & $0.88,1.24$ & 0.91 & $0.74,1.14$ & $1 \cdot 20$ & $0.90,1.60$ & 0.5640 \\
\hline Model $1 \dagger$ & 1.0 & Ref. & 1.07 & $0.88,1.32$ & 0.80 & $0.62,1.03$ & 0.97 & $0.70,1.34$ & 0.3943 \\
\hline Model $2 \ddagger$ & 1.0 & Ref. & 1.03 & $0.84,1.27$ & 0.75 & $0.58,0.97$ & 0.90 & $0.64,1.25$ & 0.1657 \\
\hline \multicolumn{10}{|l|}{ Abdominal obesity } \\
\hline No. of cases & \multicolumn{2}{|c|}{650} & \multicolumn{2}{|c|}{975} & \multicolumn{2}{|c|}{406} & \multicolumn{2}{|c|}{127} & \\
\hline Age- and sex-adjusted & 1.0 & Ref. & 1.03 & $0.89,1.18$ & 1.06 & $0.89,1.26$ & $1 \cdot 11$ & $0.86,1.43$ & 0.3845 \\
\hline Model $1 \dagger$ & $1 \cdot 0$ & Ref. & 1.00 & $0.80,1 \cdot 24$ & 0.84 & $0.64,1.10$ & 0.82 & $0.56,1.22$ & 0.2114 \\
\hline Model $2 \ddagger$ & 1.0 & Ref. & 0.97 & $0.78,1.21$ & 0.81 & $0.62,1.06$ & 0.78 & $0.53,1.16$ & 0.1156 \\
\hline \multicolumn{10}{|l|}{ Low HDL-cholesterol } \\
\hline No. of cases & \multicolumn{2}{|c|}{789} & \multicolumn{2}{|c|}{1101} & \multicolumn{2}{|c|}{426} & \multicolumn{2}{|c|}{158} & \\
\hline Age- and sex-adjusted & 1.0 & Ref. & 1.01 & $0.89,1.15$ & 1.09 & $0.92,1.28$ & 1.49 & $1 \cdot 16,1.91$ & 0.0127 \\
\hline Model $1 \dagger$ & 1.0 & Ref. & 0.99 & $0.87,1.13$ & 0.99 & $0.83,1.17$ & 1.34 & $1.03,1.75$ & 0.1806 \\
\hline Model $2 \ddagger$ & 1.0 & Ref. & 1.00 & $0.87,1.14$ & 0.99 & $0.84,1.18$ & 1.36 & $1.04,1.77$ & 0.1623 \\
\hline \multicolumn{10}{|l|}{ Hypertriacylglycerolaemia } \\
\hline No. of cases & \multicolumn{2}{|c|}{565} & \multicolumn{2}{|c|}{789} & \multicolumn{2}{|c|}{396} & & & \\
\hline Age- and sex-adjusted & 1.0 & Ref. & 1.04 & $0.90,1 \cdot 21$ & 1.03 & $0.85,1.24$ & 1.13 & $0.88,1.45$ & 0.3794 \\
\hline Model $1 \dagger$ & 1.0 & Ref. & 1.00 & $0.85,1.17$ & 0.87 & $0.71,1.07$ & 0.82 & $0.63,1.07$ & 0.0981 \\
\hline Model $2 \ddagger$ & 1.0 & Ref. & 0.99 & $0.84,1.16$ & 0.86 & $0.69,1.06$ & 0.81 & $0.62,1.05$ & 0.0766 \\
\hline Hyperglycaemia & & & & & & & & & \\
\hline No. of cases & & & & & & & & & \\
\hline Age- and sex-adjusted & 1.0 & Ref. & 0.89 & $0.76,1.04$ & 0.93 & $0.77,1.13$ & 1.00 & $0.76,1.31$ & 0.6480 \\
\hline Model $1 \dagger$ & 1.0 & Ref. & 0.88 & $0.75,1.04$ & 0.86 & $0.70,1.05$ & 0.83 & $0.62,1.11$ & 0.0992 \\
\hline Model $2 \ddagger$ & $1 \cdot 0$ & Ref. & 0.87 & $0.73,1.02$ & 0.83 & $0.68,1.01$ & 0.79 & $0.59,1.06$ & 0.0416 \\
\hline Elevated blood pressure & & & & & & & & & \\
\hline No. of cases & & & & & & & & & \\
\hline Age- and sex-adjusted & $1 \cdot 0$ & Ref. & 1.08 & $0.92,1.26$ & 0.92 & $0.75,1.14$ & 0.91 & $0.67,1.24$ & 0.4963 \\
\hline Model $1 \dagger$ & 1.0 & Ref. & $1 \cdot 12$ & $0.95,1.32$ & 0.94 & $0.76,1.17$ & 0.88 & $0.65,1.20$ & 0.4971 \\
\hline Model $2 \ddagger$ & 1.0 & Ref. & 1.11 & $0.94,1.31$ & 0.93 & $0.75,1.16$ & 0.88 & $0.65,1.20$ & 0.4727 \\
\hline
\end{tabular}

Ref., reference.
* $P$-values were derived from a regression model using the PROC SURVEYLOGISTIC procedure.

† Model 1 adjusted for age, sex, household income, education, smoking, alcohol, total energy intake, survey year, physical activity and BMI.

¥ Model 2 adjusted for age, sex, household income, education, smoking, alcohol, total energy intake, survey year, physical activity, BMI, sleep duration, intakes of soda, green tea, vegetables, red and white meat, legumes, fruit, whole grains, fish, nuts and dairy products. 
Coffee consumption and the metabolic syndrome

metabolic syndrome are required to identify adequate amount of coffee consumption for metabolic health.

In Korea, many people usually drink coffee with coffee additives such as sugar and cream ${ }^{(18)}$, and thus instant coffee mixes including sugar and cream have formed the highest proportions of the Korean coffee market ${ }^{(17)}$. Considering that cream and sugar generally could contribute to weight gain and insulin resistance ${ }^{(20,39,40)}$, high consumption of coffee with sugar and cream might have an adverse effect on metabolic health among obese individuals. In this study, we conducted a stratified analysis to investigate whether the association between coffee consumption and the metabolic syndrome varied by obesity status. As a result, we observed somewhat different associations between coffee consumption and the metabolic syndrome by obesity status. We found an inverse linear relationship between coffee intake and the metabolic syndrome among non-obese participants. On the other hand, a non-linear relationship was observed among obese participants. Compared to $<1$ time of coffee/d, 3-4 times of coffee/d was inversely associated with the metabolic syndrome, and five or more times of coffee per day was not significantly associated with the metabolic syndrome, showing a non-significant positive relationship. The inverse association, which was found among obese people who consumed 3-4 times of coffee/d, became slightly stronger when we further adjusted for amount of coffee additives such as cream and sugar $(\mathrm{OR}=0.64,95 \% \mathrm{CI}$ $0.44,0.91)$. This change by adjustment for coffee additives might suggest the possibility that the inverse association between coffee intake and the metabolic syndrome was attenuated due to high intake of sugar and cream among obese people. The difference in effects of added sugar intake according to obesity status was also found in previous studies. The results from Canadian cohort showed a positive association between high intake of added sugars from liquid and components of the metabolic syndrome among overweight subjects but not normal-weight subjects ${ }^{(22)}$. A cross-sectional study from nationally representative data of USA reported that higher consumption of added sugars was associated with increased insulin resistance only in overweight participants ${ }^{(21)}$.

Our study has several limitations. First, the current study is a cross-sectional design and thus it is hard to determine a causal association between coffee consumption and the metabolic syndrome. To reduce reverse causation bias, we excluded subjects taking medication to treat diabetes, hypertension or dyslipidaemia, or having diseases such as diabetes, hypertension, dyslipidaemia, myocardial infarction, stroke or cancer. Second, there is a possibility of misclassification because information of coffee intake was obtained using FFQ. However, the misclassification of coffee consumption tends to underrate, rather than exaggerate, the results by diluting the association because it is non-differential and can only make the exposure groups more similar. Lastly, BMI is not the best indicator of obesity although we defined obesity by BMI, and our results from stratified analysis by obesity status should be interpreted with caution because the number of cases is relatively small. Despite of these limitations, the results of our study can be applied to the general healthy Korean adult populations because we analysed using the data from nationally 
representative surveys. We selected the study population more rigorously by excluding the subjects with diseases than previous studies that investigated the relationship between coffee and the metabolic syndrome in Koreans. To reduce confounding problems inherent in observational studies, we controlled for dietary intakes of many food groups as well as health behaviours such as smoking and alcohol consumption. Our results were different from those of previous studies which explored the relation of coffee consumption and the metabolic syndrome among Korean adults ${ }^{(13-15)}$ in that we suggested that moderate coffee consumption of 3-4 times/d was associated with the metabolic syndrome. The differences in results might be due to selection of the study population by rigorous criteria and adjustment for dietary intakes of many food groups in the analyses. Finally, to our knowledge, this is the first study which examined the association between coffee consumption and the metabolic syndrome by obesity status.

In conclusion, moderate coffee consumption of 3-4 times/d was inversely associated with the metabolic syndrome among Korean adult population. The association between heavy coffee consumption more than 5 times/d and the metabolic syndrome was different among obese- and non-obese participants. Further randomised controlled trials and well-designed prospective studies should be conducted in large populations to identify adequate amount of coffee consumption for metabolic health.

\section{Supplementary material}

For supplementary material/s referred to in this article, please visit https://doi.org/10.1017/S0007114518002635

\section{Acknowledgements}

The authors thank all staff and study participants of KNHANES.

This work was supported by the Basic Science Research Program through the National Research Foundation of Korea, funded by the Ministry of Science, ICT and Future Planning (NRF-2015R1A1A1A05001362) and by the Ministry of Education (NRF-2017R1A6A3A01075728). Funders had no role in the study design, data collection and analysis, decision to publish or preparation of the manuscript.

The authors' contributions were as follows: Y. J. developed study concept and design and contributed to critical revision of the manuscript for important intellectual content; Y. K. wrote the draft of manuscript, conducted statistical analyses and had primary responsibility for the final content; Y. K. and Y. J. contributed to discussion and reviewed/edited the manuscript. All authors have read and approved the final version submitted for publication.

The authors declare that there are no conflicts of interest.

\section{References}

1. Eckel RH, Grundy SM \& Zimmet PZ (2005) The metabolic syndrome. Lancet 365, 1415-1428.

2. Esposito K, Chiodini P, Colao A, et al. (2012) Metabolic syndrome and risk of cancer: a systematic review and metaanalysis. Diabetes Care 35, 2402-2411.
3. Alberti KG, Eckel RH, Grundy SM, et al. (2009) Harmonizing the metabolic syndrome: a joint interim statement of the International Diabetes Federation Task Force on Epidemiology and Prevention; National Heart, Lung, and Blood Institute; American Heart Association; World Heart Federation; International Atherosclerosis Society; and International Association for the Study of Obesity. Circulation 120, 1640-1645.

4. Galassi A, Reynolds K \& He J (2006) Metabolic syndrome and risk of cardiovascular disease: a meta-analysis. Am J Med $\mathbf{1 1 9}$, 812-819.

5. Kolovou GD, Anagnostopoulou KK, Salpea KD, et al. (2007) The prevalence of metabolic syndrome in various populations. Am J Med Sci 333, 362-371.

6. Lim S, Shin H, Song JH, et al. (2011) Increasing prevalence of metabolic syndrome in Korea: the Korean National Health and Nutrition Examination Survey for 1998-2007. Diabetes Care 34, 1323-1328.

7. Kim Y \& Je Y (2018) Meat consumption and risk of metabolic syndrome: results from the Korean population and a metaanalysis of observational studies. Nutrients 10, E390.

8. Narain A, Kwok CS \& Mamas MA (2017) Soft drink intake and the risk of metabolic syndrome: a systematic review and metaanalysis. Int J Clin Pract 71, e12927.

9. Shang F, Li X \& Jiang X (2016) Coffee consumption and risk of the metabolic syndrome: a meta-analysis. Diabetes Metab $\mathbf{4 2}$, $80-87$.

10. Marventano S, Salomone F, Godos J, et al. (2016) Coffee and tea consumption in relation with non-alcoholic fatty liver and metabolic syndrome: a systematic review and meta-analysis of observational studies. Clin Nutr 35, 1269-1281.

11. Kim Y \& Je Y (2016) Dairy consumption and risk of metabolic syndrome: a meta-analysis. Diabetes Med 33, 428-440.

12. Yesil A \& Yilmaz Y (2013) Review article: coffee consumption, the metabolic syndrome and non-alcoholic fatty liver disease. Aliment Pharmacol Ther 38, 1038-1044.

13. Kim K, Kim K \& Park SM (2016) Association between the prevalence of metabolic syndrome and the level of coffee consumption among Korean women. PLOS ONE 11, e0167007.

14. Kim HJ, Cho S, Jacobs DR Jr, et al. (2014) Instant coffee consumption may be associated with higher risk of metabolic syndrome in Korean adults. Diabetes Res Clin Pract 106, 145-153.

15. Shin H, Linton JA, Kwon Y, et al. (2017) Relationship between coffee consumption and metabolic syndrome in Korean adults: data from the 2013-2014 Korea National Health and Nutrition Examination Survey. Korean J Fam Med 38, 346-351.

16. Ministry of Health and Welfare, Korea Centers for Disease Control and Prevention (2017) Korea Health Statistics 2016: Korea National Health and Nutrition Examination Survey (KNHANES VII-1). Cheongju: Korea Centers for Disease Control and Prevention.

17. USDA Foreign Agricultural Service (2015) Coffee Market Brief Update-Seoul ATO; Global Agricultural Information Network Report. Washington, DC: United States Department of Agriculture Foreign Agricultural Service.

18. Je Y, Jeong S \& Park T (2014) Coffee consumption patterns in Korean adults: the Korean National Health and Nutrition Examination Survey (2001-2011). Asia Pac J Clin Nutr 23, 691-702.

19. Lin TK, Teymourian Y \& Tursini MS (2018) The effect of sugar and processed food imports on the prevalence of overweight and obesity in 172 countries. Global Health 14, 35.

20. Mancini A, Imperlini E, Nigro E, et al. (2015) Biological and nutritional properties of palm oil and palmitic acid: effects on health. Molecules 20, 17339-17361. 
21. Welsh JA, Sharma A, Cunningham SA, et al. (2011) Consumption of added sugars and indicators of cardiovascular disease risk among US adolescents. Circulation 123, 249-257.

22. Wang JW, Mark S, Henderson M, et al. (2013) Adiposity and glucose intolerance exacerbate components of metabolic syndrome in children consuming sugar-sweetened beverages: QUALITY cohort study. Pediatr Obes 8, 284-293.

23. Kweon S, Kim Y, Jang MJ, et al. (2014) Data resource profile: the Korea National Health and Nutrition Examination Survey (KNHANES). Int J Epidemiol 43, 69-77.

24. Kim DW, Song S, Lee JE, et al. (2015) Reproducibility and validity of an FFQ developed for the Korea National Health and Nutrition Examination Survey (KNHANES). Public Health Nutr 18, 1369-1377.

25. Shin HJ, Cho E, Lee HJ, et al. (2014) Instant noodle intake and dietary patterns are associated with distinct cardiometabolic risk factors in Korea. $J$ Nutr $\mathbf{1 4 4}, 1247-1255$.

26. Lee HH, Lee HJ, Cho JI, et al. (2013) Overall and abdominal adiposity and hypertriglyceridemia among Korean adults: the Korea National Health and Nutrition Examination Survey 2007-2008. Eur J Clin Nutr 67, 83-90.

27. Lutsey PL, Steffen LM \& Stevens J (2008) Dietary intake and the development of the metabolic syndrome: the Atherosclerosis Risk in Communities study. Circulation 117, 754-761.

28. Baik I, Lee M, Jun NR, et al. (2013) A healthy dietary pattern consisting of a variety of food choices is inversely associated with the development of metabolic syndrome. Nutr Res Pract 7, 233-241.

29. Ding M, Bhupathiraju SN, Satija A, et al. (2014) Long-term coffee consumption and risk of cardiovascular disease: a systematic review and a dose-response meta-analysis of prospective cohort studies. Circulation 129, 643-659.

30. Grosso G, Micek A, Godos J, et al. (2016) Coffee consumption and risk of all-cause, cardiovascular, and cancer mortality in smokers and non-smokers: a dose-response meta-analysis. Eur J Epidemiol 31, 1191-1205.
31. Je Y \& Giovannucci E (2014) Coffee consumption and total mortality: a meta-analysis of twenty prospective cohort studies. Br J Nutr 111, 1162-1173.

32. Grosso G, Godos J, Galvano F, et al. (2017) Coffee, caffeine, and health outcomes: an umbrella review. Annu Rev Nutr 37, 131-156.

33. Grosso G, Marventano S, Galvano F, et al. (2014) Factors associated with metabolic syndrome in a Mediterranean population: role of caffeinated beverages. I Epidemiol $\mathbf{2 4}$, 327-333.

34. Ong KW, Hsu A \& Tan BK (2013) Anti-diabetic and antilipidemic effects of chlorogenic acid are mediated by AMPK activation. Biochem Pharmacol 85, 1341-1351.

35. Arion WJ, Canfield WK, Ramos FC, et al. (1997) Chlorogenic acid and hydroxynitrobenzaldehyde: new inhibitors of hepatic glucose 6-phosphatase. Arch Biochem Biophys 339, 315-322.

36. Suzuki A, Yamamoto M, Jokura H, et al. (2007) Ferulic acid restores endothelium-dependent vasodilation in aortas of spontaneously hypertensive rats. Am J Hypertens 20, 508-513.

37. Cho AS, Jeon SM, Kim MJ, et al. (2010) Chlorogenic acid exhibits anti-obesity property and improves lipid metabolism in high-fat diet-induced-obese mice. Food Chem Toxicol 48, 937-943.

38. Moreira AS, Nunes FM, Domingues MR, et al. (2012) Coffee melanoidins: structures, mechanisms of formation and potential health impacts. Food Funct 3, 903-915.

39. Siervo M, Montagnese C, Mathers JC, et al. (2014) Sugar consumption and global prevalence of obesity and hypertension: an ecological analysis. Public Health Nutr 17, 587-596.

40. Wang J, Light K, Henderson M, et al. (2014) Consumption of added sugars from liquid but not solid sources predicts impaired glucose homeostasis and insulin resistance among youth at risk of obesity. J Nutr 144, 81-86. 